\title{
Uso do Sistema de Classificação de Robson na avaliação das taxas de cesariana em Santa Catarina e sua associação com perfil institucional
}

\section{Use of Robson Classification to assess cesarean section rates in Santa Catarina and its association with source of payment for childbirth}

\author{
Paulo Fontoura Freitas ${ }^{1}$ (D) , Helena Gondin May Vieira ${ }^{2}(\mathbb{0}$ \\ 1. Docente do curso de Medicina pela Universidade do Sul de Santa Catarina (UNISUL) - Pedra Branca, Palhoça, SC, Brasil. 2. Discente do curso de Medicina \\ pela Universidade do Sul de Santa Catarina (UNISUL) - Pedra Branca, Palhoça, SC, Brasil
}

\begin{abstract}
Resumo
Objetivo: analisar as diferenças nas taxas de cesariana em Santa Catarina, ao comparar os perfis público e privado, de acordo com grupos do Sistema de Classificação de Robson. Métodos: estudo transversal baseado no Sistema de Informação dos Nascidos Vivos de Santa Catarina (SINASC/SC), 2012. Foram calculadas as taxas globais e específicas de cesariana de acordo com o grupo do SCR, além do excesso de cesarianas pelo Risco Atribuível Proporcional (RAP). Diferenças nas proporções de partos e taxas de cesariana em cada grupo, de acordo com a forma de pagamento, foram analisadas pelo teste do qui-quadrado, a 5\% de significância. Resultados: a taxa global de cesariana foi de $60,7 \%$, sendo 88,9\% no sistema privado e $45,7 \%$ no setor público. Os grupos 1, 4 e 5 do SCR tiveram maior impacto nas taxas globais. No setor privado, chamou à atenção a baixa utilização da indução, com mais de $65 \%$ dos partos ocorrendo eletivamente, além da alta concentração de multíparas com cesariana prévia. Conclusão: taxas elevadas entre primíparas antecipam o efeito cumulativo da cesariana prévia em multíparas. A grande proporção de cesarianas eletivas em detrimento do manejo ativo do trabalho de parto, principalmente no setor privado, aponta para um grande número de cesarianas realizadas sem indicações médicas e preocupam devido a potenciais efeitos adversos para mães e recém-nascidos.
\end{abstract}

Palavras-chave: Cesárea. Brasil. Classificação. Sistemas de Saúde.

\begin{abstract}
Objective: to analyze cesarean section rates in Santa Catarina and its association with source of payment for childbirth. Methods: Cross-sectional study based on the Information System of Live Births of Santa Catarina (SINASC/SC), 2012. The global and specific cesarean rates were calculated according to the SCR groups, in addition to the excess of cesarean sections due to the Proportional Attributable Risk (RAP). Differences in birth proportions and cesarean section in each group, according to the form of payment, were analyzed by the chi-square test at $5 \%$ significance level. Results: The overall cesarean section rate was $60.7 \%$, being $88.9 \%$ in the private system and $45.7 \%$ in the public one. Groups 1,4 and 5 of RCS were the ones with the greatest impact on cesarean overall rates. In the private sector, attention was drawn to the low use of induction, with more than $65 \%$ of deliveries occurring electively, in addition to the high concentration of multiparous women with previous cesarean section. Conclusions: High rates among primiparous women anticipate the cumulative effect of previous cesarean section in multiparous women. The large proportion of elective cesareans sections at the expense of active labor management, especially in the private sector, points to a large number of cesarean sections performed without medical indications and concerns due to potential adverse effects on mothers and newborns.
\end{abstract}

Keywords: Cesarean section. Brazil. Classification. Health System.

\section{INTRODUÇÃO}

O aumento das taxas de parto cesáreo é um fenômeno mundial $^{1}$. No Brasil, a proporção de nascimentos por cesariana aumentou de 41,7\% em 2004 para 56,6\% em 2014. Em Santa Catarina, nesse mesmo ano, $60 \%$ dos nascimentos se deram por essa via de parto ${ }^{2}$. Esses índices encontram-se acima do estabelecido pela Organização Mundial da Saúde $(O M S)^{3}$ e preocupam devido à potencial associação com o aumento no risco de morbimortalidade para a mãe e o recém-nascido ${ }^{4,5,6}$.

Diferenças no uso do parto cesáreo estão, fortemente, associadas à fonte de pagamento pelo parto, com taxas bem mais elevadas no setor privado, quando comparadas ao setor público $^{7,8}$. Nesse contexto, tem sido postulado que, no Brasil, uma importante parte das cesarianas é realizada sem indicações clínicas suficientes ${ }^{9}$.

Diversos estudos sugerem que a decisão pela via de parto reflete condições socioculturais e da prática obstétrica ${ }^{6}$, assim como fatores institucionais e geográficos, financeiros, étnicos e legais ${ }^{9,10,11}$. Diferenças significativas nas taxas de cesárea, entre países, regiões de um mesmo país e entre instituições em uma mesma população ${ }^{1}$, apontam para a necessidade de uma classificação que permita maior comparabilidade entre grupos específicos $^{12}$.

Correspondente: Paulo Fontoura Freitas. Universidade do Sul de Santa Catarina (UNISUL) - Pedra Branca. Núcleo de Orientação em Epidemiologia. Curso de Medicina. Rua Beco da Lua, 232, Lagoa da Conceição, CEP: 88062-490, Florianópolis, SC, Brasil. E-mail: pfreitas.epidemio@gmail.com

Conflito de interesse: Não há conflito de interesse por parte de qualquer um dos autores.

Recebido em: 23 Maio 2019; Revisado em: 5 Jul 2019; 24 Out 2019; Aceito em: 6 Nov 2019 
Em 2011, uma revisão sistemática de diferentes métodos de classificação, conduzida pela Organização Mundial da Saúde $(\mathrm{OMS})^{13}$, definiu o Sistema de Classificação de Robson (SCR) como padrão global no monitoramento e na comparação das taxas de cesariana ${ }^{3}$. Baseado em parâmetros obstétricos objetivos, o SCR distribui as parturientes em dez grupos totalmente inclusivos e mutuamente exclusivos, de acordo com paridade, início do trabalho de parto, idade gestacional, apresentação fetal e número de fetos. A flexibilidade dessa classificação permite melhor entendimento das práticas obstétricas locais e da contribuição específica de cada grupo, permitindo estratégias de saúde pública direcionadas para a redução do parto cesáreo ${ }^{14}$.

Nos últimos anos, o SCR tem sido extensivamente utilizado na avaliação dos determinantes do parto por via cirúrgica ao comparar diferentes instituições, regiões e países ${ }^{15}$. Em um desses estudos, a OMS realizou uma análise secundária baseada em dois inquéritos e utilizou a classificação de Robson no entendimento das taxas de cesárea em 21 países $^{16}$.

O presente estudo parte do pressuposto de que diferenças nas taxas de parto cesáreo em Santa Catarina, ao comparar grupos de parturientes com base no Sistema de Classificação de Robson, sejam mediadas pelo perfil institucional, aqui representado pelos sistemas público e privado de assistência ao parto, e justifica sua importância pela ausência de estudos no objeto específico. O objetivo central se refere à exploração de diferenças nas taxas de cesariana, ao comparar os perfis público e privado de acordo com grupos do Sistema de Classificação de Robson.

\section{MÉTODOS}

\section{Fonte dos dados e amostra}

Trata-se de um estudo com delineamento transversal baseado na informação a respeito dos eventos da gestação e do parto, para os nascidos vivos no estado de Santa Catarina, no ano de 2012, obtida a partir do banco de dados do Sistema de Informação de Nascidos Vivos (SINASC) para Santa Catarina, disponibilizado online pelo Ministério da Saúde ${ }^{17}$. Foram excluídos os partos não hospitalares, por não estarem em risco de cesariana. Foram selecionados, em cada uma das nove macrorregionais do estado, os seis municípios com maior número de partos. Para cada um desses municípios, foram considerados todos os estabelecimentos que possuíam leitos obstétricos/ maternidade. Uma amostra final de 61 maternidades, com um total de 56.632 partos, foi calculada como representativa das taxas de cesariana para o estado de Santa Catarina como um todo $(p<0,001)$. Informações a respeito do perfil de cada uma das maternidades estudadas foram obtidas por meio do Cadastro Nacional de Estabelecimentos de Saúde $(C N E S)^{18}$. As maternidades selecionadas foram classificadas em três grupos: públicas, privadas e mistas. Essa classificação teve como base de cálculo a "razão entre partos públicos e privados", obtida pela divisão entre o número de leitos públicos e privados, utilizando a informação fornecida pelo CNES. Assim, foram classificadas como públicas aquelas maternidades com $100 \%$ de leitos do Sistema Único de Saúde (SUS) e, como privadas, aquelas com $100 \%$ de leitos privados. Por sua vez, as maternidades classificadas como "mistas" combinavam diferentes proporções de leitos públicos e privados.

\section{Grupos no SCR e covariáveis}

O desfecho foi definido na forma dicotômica via de parto (1=cesariana; 2=vaginal). As variáveis independentes encontram-se representadas por cada um dos 10 grupos do Sistema de Classificação de Robson. As variáveis utilizadas pelo SCR, para classificação das mulheres em cada um dos 10 grupos, incluem paridade (primíparas ou multíparas: com e sem cesariana prévia), início do trabalho de parto (espontâneo, induzido ou cesariana eletiva), idade gestacional (abaixo de 37 semanas e 37 ou mais semanas de gestação), apresentação fetal (cefálica, pélvica ou transversa) e número de fetos (único ou múltiplos).

Além das variáveis componentes do SCR, foram obtidas aquelas que representam características socioeconômicas, demográficas, reprodutivas e institucionais: idade materna (em anos: 10-14; 15-19; 20-34; 35 e +), escolaridade materna (em anos de escolaridade: 0-3; 4-7; 8-11; 12 e +), etnia/cor da pele (branca; não branca), estado civil (casada/união estável; solteira; outros), número de consultas pré-natal (0-6; 7 e +) e perfil institucional (público; privado; misto).

\section{Análise estatística}

Os dados coletados a partir do SINASC foram exportados para o programa Statistical Package for the Social Sciences (SPSS) versão 18.0 (SPSS Inc., Chicago, IL, USA), no qual foram conduzidas as análises estatísticas. Primeiramente, foram descritas a distribuição de todos os partos, de acordo com os grupos do SCR, a taxa global e as taxas específicas de cesariana, de acordo com o grupo do SCR e como proporção do total de cesarianas. A seguir, com o objetivo de analisar os fatores associados ao excesso no número e na proporção de cesarianas, foram obtidas as Razões de Prevalência (RP), o Risco Atribuível Proporcional (RAP) e o Excesso Absoluto (EA) no número de partos cesáreos, ao comparar grupos específicos detectados anteriormente pelo SCR como de risco para cesariana (parto induzido, apresentação não cefálica, gestações múltiplas, idade gestacional pré-termo e cesariana prévia), estratificados por paridade. Foi ainda descrita a distribuição das mulheres de acordo com características maternas: socioeconômicas, demográficas e reprodutivas, de acordo com forma de pagamento pelo parto (sistemas público ou privado). Finalmente, foi apresentada a distribuição dos partos e das taxas de cesariana, como proporção de todos os partos, de acordo com o SCR, estratificados por forma de pagamento pelo parto. Diferenças nas proporções de partos em cada um dos grupos e das taxas de cesariana, de acordo com a forma de pagamento, foram analisadas pelo teste do qui-quadrado de Pearson, a um nível de significância pré-estabelecido de $<5 \%$. 
Considerações Éticas

O presente estudo foi aprovado pelo Comitê de Ética em Pesquisa com Seres Humanos da Universidade do Sul de Santa Catarina (CEP/UNISUL - CAAE: 70220617.9.0000.5369).

\section{RESULTADOS}

A tabela 1 apresenta a distribuição geral dos partos e das taxas de cesariana em Santa Catarina, no ano de 2012, de acordo com o SCR. A taxa geral de cesáreas, em Santa Catarina, no ano estudado, foi de $60,7 \%$. Foi visto que $57,9 \%$ dos partos ocorreram entre mulheres dos grupos 2, 4 e 5, e esses três grupos combinados foram responsáveis por $67,4 \%$ das cesarianas. Por outro lado, os grupos 1 e 3 perfizeram 25,5\% de todos os partos, sendo responsáveis por apenas $14 \%$ das cesarianas e com uma taxa média de cesarianas de metade daquela encontrada nos grupos 2 , 4 e 5 (33,5\% versus $67,8 \%$; $p<0,01)$.

Tabela 1. Distribuição dos partos de acordo com as variáveis do Sistema de Classificação de Robson (SCR) e taxas de cesariana de acordo com grupo e como proporção do total de cesarianas. Santa Catarina, 2012.

\begin{tabular}{|c|c|c|c|c|c|c|c|}
\hline \multirow{2}{*}{\multicolumn{2}{|c|}{ GRUPOS DO SCR }} & \multicolumn{2}{|c|}{ Todos os partos } & \multirow{2}{*}{$\begin{array}{c}\text { Cesarianas } \\
\mathrm{n}\end{array}$} & \multicolumn{3}{|c|}{ Como $\%$ das cesarianas } \\
\hline & & $\mathbf{n}$ & (\%) & & (\%) & $\mathbf{n}$ & (\%) \\
\hline 1 & (Pri; Un; Cf; $\geq 37 ;$ Esp) & 7111 & 12,6 & 3461 & 48,7 & 3461 & 10,1 \\
\hline 2 & (Pri; Un; Cf; $\geq 37 ;$ Ind/El) & 13317 & 23,5 & 9417 & 70,7 & 9417 & 27,4 \\
\hline 3 & (Mt; Un; Cf; $\geq 37 ;$ Esp; Scp) & 7328 & 12,9 & 1340 & 18,3 & 1340 & 3,9 \\
\hline 4 & (Mt; Un; Cf; $\geq 37 ;$ Ind/El; Scp) & 7746 & 13,7 & 3539 & 45,7 & 3539 & 10,3 \\
\hline 5 & (Mt; Un; Cf; $\geq 37 ;$ Ccp) & 11747 & 20,7 & 10214 & 87,0 & 10214 & 29,7 \\
\hline 6 & (Pri; Un; Pv) & 1011 & 1,8 & 915 & 90,5 & 915 & 2,7 \\
\hline 7 & (Mt; Un; Pv) & 1140 & 2,0 & 972 & 85,3 & 972 & 2,8 \\
\hline 8 & (Todas; Mtp) & 1276 & 2,3 & 1099 & 86,2 & 1099 & 3,2 \\
\hline 9 & (Todas; Un; Tv/Ob) & 146 & 0,3 & 140 & 95,9 & 140 & 0,4 \\
\hline 10 & (Todas; Un; Cf; <37) & 5810 & 10,3 & 3274 & 56,4 & 3274 & 9,5 \\
\hline \multicolumn{2}{|c|}{ Total } & 56632 & 100,0 & 34371 & 60,7 & 34371 & 100,0 \\
\hline
\end{tabular}

Legenda: Pri (primípara); Mt (multípara); Un (gestação única); Mtp (gestação múltipla); Cf (cefálica); Pv (pélvica); Tv/Ob (transversa ou oblíqua); Ind/El (indução ou cesariana eletiva); Esp (espontâneo); <37(menos de 37 semanas de gestação); $\geq 37$ (37 ou mais semanas de gestação); Scp (sem cesariana prévia); Ccp (com cesariana prévia); todas (todas as mulheres).

As taxas de cesariana entre as primíparas do grupo 1 foram mais que o dobro quando comparadas às multíparas com as mesmas características, pertencentes ao grupo 3 (48,7\% versus $18,3 \%$, respectivamente; $p<0,01)$. As mulheres nos grupos 6,7 , 8 e 9 foram responsáveis por apenas $6,4 \%$ dos partos e $9,1 \%$ de todas as cesarianas. Por fim, o grupo 10 , compreendido pelas mulheres com recém-nascidos prematuros, foi responsável por $10,3 \%$ dos partos e apresentou uma taxa de cesariana relativamente alta, 56,4\%.

Achados apresentados na tabela 2 comparam os grupos expostos e não expostos a fatores de risco em busca do excesso nas taxas de cesariana que poderia ser atribuído a cada um dos grupos.

Os resultados mostram que as taxas de cesariana foram acima de $70 \%$ para quase todos os grupos de risco do SCR, sendo, especialmente, altas entre as mulheres cujos fetos tiveram apresentações não cefálicas, tanto entre as primíparas $(R P=1,63 ; p<0,001)$ quanto entre as multíparas sem cesárea prévia, com feto único e pélvico, tendo parto espontâneo e a termo $(R P=4,09 ; p<0,001)$, quando comparadas àquelas dos mesmos grupos, com parto cefálico.
Além disso, multíparas com gestação múltipla e sem cesárea prévia apresentaram taxas cinco vezes mais elevadas quando comparadas àquelas do mesmo grupo com feto único (RP= $5,04 ; p<0,001)$. Ainda que as multíparas com gestação múltipla e com cesárea prévia tenham apresentado taxas de cesariana iguais ao grupo sem cesárea prévia (93\%), a diferença quando comparadas àquelas do mesmo grupo com parto único, não foi, significativamente, relevante $(R P=1,21 ; p=0,21)$.

O Risco Atribuível Percentual (RAP), o qual mede a contribuição no excesso de cesarianas que pode ser atribuído a grupos específicos (expostos), indica que foi encontrado um excesso absoluto de 13,8\%, ou seja, 6.402 de um total de 46.471 cesarianas ocorreram como contribuição dos grupos de risco (expostos) como um todo.

Mulheres com parto induzido e/ou cesariana eletiva foram responsáveis por quase $80 \%$ do excesso no número de cesarianas, $45,77 \%$ e 33,51\%, respectivamente, e, juntamente com cesariana prévia, com $16,39 \%$, somaram mais de $95 \%$ do excesso absoluto no número de cesarianas comparado aos demais grupos de risco combinados, os quais chegaram a menos de $5 \%$ do excesso relativo $(p<0,001)$. 
Tabela 2. Razões de prevalência (RP); risco atribuível proporcional (RAP) e excesso absoluto (En) e proporcional (E\%) no número de partos cesáreos ao comparar grupos específicos de risco no Sistema de Classificação de Robson. Santa Catarina, 2012.

\begin{tabular}{|c|c|c|c|c|c|c|c|}
\hline \multirow[b]{2}{*}{ Grupos (SCR) } & \multicolumn{7}{|c|}{ Partos cesáreos } \\
\hline & № & (\%) & RP & RAP \% & $\begin{array}{r}\text { Excesso } \\
\text { (En) }\end{array}$ & $\begin{array}{r}\text { Excesso } \\
\text { (E\%) }\end{array}$ & p-valor \\
\hline \multicolumn{8}{|l|}{ Pr; Un, Cf; $\geq 37$} \\
\hline Induzido & 9390 & 70,7 & 1,45 & 31,2 & 2930 & 45,77 & $<0,001$ \\
\hline Espontâneo & 3443 & 48,6 & & & & & \\
\hline \multicolumn{8}{|l|}{ Mt; Scp, Un, Cf; $\geq 37$} \\
\hline Induzido & 3611 & 45,6 & 2,46 & 59,4 & 2145 & 33,51 & $<0,001$ \\
\hline Espontâneo & 1383 & 18,5 & & & & & \\
\hline \multicolumn{8}{|l|}{ Mt; Ccp; $\geq 37$; Esp } \\
\hline Feto único pélvico & 131 & 80,4 & 1,05 & 4,4 & 6 & 0,09 & 0,29 \\
\hline Feto único cefálico & 3029 & 76,8 & & & & & \\
\hline \multicolumn{8}{|l|}{ Mt; Scp; $\geq 37$; Esp } \\
\hline Feto único pélvico & 160 & 75,8 & 4,09 & 75,5 & 121 & 1,89 & $<0,001$ \\
\hline Feto único cefálico & 1383 & 18,5 & & & & & \\
\hline \multicolumn{8}{|l|}{ Pr; $\geq 37$; Esp } \\
\hline Feto único pélvico & 274 & 83,8 & 1,63 & 42,0 & 115 & 1,80 & $<0,001$ \\
\hline Feto único cefálico & 3443 & 48,6 & & & & & \\
\hline \multicolumn{8}{|l|}{ Mt; Scp, Cf; $\geq 37$; Esp } \\
\hline Gestação múltipla & 14 & 93,3 & 5,04 & 80,1 & 11 & 0,17 & 0,21 \\
\hline Gestação única & 1383 & 18,5 & & & & & \\
\hline \multicolumn{8}{|l|}{ Mt; Ccp; \37; Esp } \\
\hline Gestação múltipla & 14 & 93,3 & 1,21 & 17,6 & 3 & 0,05 & 0,21 \\
\hline Gestação única & 3029 & 76,8 & & & & & \\
\hline \multicolumn{8}{|l|}{ Pri; Un; $\geq 37$; Esp } \\
\hline Gestação múltipla & 9 & 81,8 & 1,27 & 40,5 & 4 & 0,06 & 0,05 \\
\hline Gestação única & 3443 & 48,6 & & & & & \\
\hline \multicolumn{8}{|l|}{ Mt; Un; Ccp; Esp } \\
\hline Apresentação transversa ou oblíqua & 17 & 94,4 & 1,23 & 18,6 & 3 & 0,05 & 0,11 \\
\hline Apresentação cefálica & 3029 & 76,8 & & & & & \\
\hline \multicolumn{8}{|l|}{ Todas; Un; Scp; Esp } \\
\hline Apresentação transversa ou oblíqua & 7 & 87,5 & 4,73 & 78,8 & 6 & 0,09 & $<0,001$ \\
\hline Apresentação cefálica & 1383 & 18,5 & & & & & \\
\hline \multicolumn{8}{|l|}{ Todas; Un; Scp; Esp } \\
\hline Idade gestacional pré-termo & 455 & 78,4 & 1,02 & 2,0 & 9 & 0,14 & 0,38 \\
\hline Idade gestacional a termo & 3029 & 76,8 & & & & & \\
\hline \multicolumn{8}{|l|}{ Mt; Un; Cf; 237; Esp } \\
\hline com cesárea prévia & 1383 & 76,8 & 4,15 & 75,9 & 1049 & 16,39 & $<0,001$ \\
\hline sem cesárea prévia & 3029 & 18,5 & & & & & \\
\hline
\end{tabular}

Pri (primípara); Mt (multípara); Un (gestação única; Cf (cefálica); Esp (espontâneo); 237 (37 ou mais semanas de gestação); Scp (sem cesariana prévia); Ccp (com cesariana prévia); todas (todas as mulheres). 
A tabela 3 apresenta as características das mulheres conforme maternidades com 100\% dos leitos obstétricos públicos ou a forma de pagamento pelo parto, de acordo com hospitais/ privados.

Tabela 3. Características das mulheres de acordo com forma de pagamento pelo parto. Santa Catarina, 2012.

\begin{tabular}{|c|c|c|c|c|c|c|c|}
\hline \multirow{2}{*}{ Variáveis } & \multicolumn{2}{|c|}{ Total } & \multicolumn{2}{|c|}{ Público } & \multicolumn{2}{|c|}{ Privado } & \multirow{2}{*}{ p-valor } \\
\hline & $\mathbf{n}$ & $\%$ & $\mathbf{n}$ & $\%$ & $\% n$ & $\%$ & \\
\hline \multicolumn{8}{|l|}{ Idade (anos) } \\
\hline$<20$ & 3324 & 14,4 & 2942 & 24,4 & 382 & 3,5 & $<0,001$ \\
\hline $20-34$ & 16655 & 72,2 & 7906 & 65,6 & 8749 & 79,3 & \\
\hline$>34$ & 3104 & 13,4 & 1209 & 10,0 & 1895 & 17,2 & \\
\hline \multicolumn{8}{|l|}{ Cor da Pele } \\
\hline Branca & 19680 & 91,5 & 9513 & 88,4 & 10167 & 94,7 & $<0,001$ \\
\hline Não branca & 1826 & 8,5 & 1253 & 11,6 & 573 & 5,3 & \\
\hline \multicolumn{8}{|l|}{ Estado Civil } \\
\hline Casada/União estável & 14508 & 63,1 & 5939 & 49,5 & 8569 & 77,8 & $<0,001$ \\
\hline Outras & 8493 & 36,9 & 6048 & 50,5 & 2445 & 22,2 & \\
\hline \multicolumn{8}{|l|}{ Escolaridade (anos) } \\
\hline$<7$ & 4255 & 18.5 & 3721 & 31,1 & 534 & 4,8 & $<0,001$ \\
\hline $8-12$ & 11728 & 51,0 & 7136 & 59,7 & 4592 & 41,7 & \\
\hline$>12$ & 6991 & 30,4 & 1106 & 9,2 & 5885 & 53,4 & \\
\hline \multicolumn{8}{|l|}{ Paridade } \\
\hline Primípara & 11215 & 48,6 & 5412 & 44,9 & 5803 & 52,6 & $<0,001$ \\
\hline Multípara & 11868 & 51,4 & 6645 & 55,1 & 5223 & 47,4 & \\
\hline \multicolumn{8}{|l|}{ Gestação múltipla } \\
\hline Sim & 423 & 1,8 & 212 & 1,7 & 211 & 1,9 & \\
\hline Não & 23083 & 98,2 & 12057 & 98,3 & 11026 & 98,1 & 0,41 \\
\hline \multicolumn{8}{|l|}{ Cesárea eletiva } \\
\hline Sim & 9708 & 43,0 & 2663 & 22,5 & 7045 & 65,4 & $<0,001$ \\
\hline Não & 12879 & 57,0 & 9157 & 77,5 & 3722 & 36,6 & \\
\hline \multicolumn{8}{|l|}{ Indução } \\
\hline Sim & 4495 & 19,9 & 3539 & 30,0 & 956 & 8,9 & $<0,001$ \\
\hline Não & 13907 & 80,1 & 8420 & 70,0 & 9842 & 91,1 & \\
\hline \multicolumn{8}{|l|}{ Cesárea prévia } \\
\hline Sim & 5492 & 24,7 & 2490 & 21,2 & 3002 & 28,7 & $<0,001$ \\
\hline Não & 16728 & 75,3 & 9259 & 78,8 & 7469 & 71,3 & \\
\hline \multicolumn{8}{|l|}{ Pré-natal ( $n \cong$ consultas) } \\
\hline$\leq 6$ & 7224 & 31,4 & 4816 & 40,1 & 2408 & 21,9 & $<0,001$ \\
\hline$\geq 7$ & 15769 & 68,6 & 7202 & 59,9 & 8567 & 78,1 & \\
\hline \multicolumn{8}{|l|}{ Tipo de parto } \\
\hline Cesariana & 15612 & 66,5 & 5594 & 45,7 & 10018 & 89,2 & $<0,001$ \\
\hline Vaginal & 7869 & 33,5 & 6652 & 54,3 & 1217 & 10,8 & \\
\hline
\end{tabular}

Porcentagem de dados que não puderam ser obtidos para as seguintes variáveis: idade $1,80 \%$, cor de pele $8,51 \%$, estado civil $2,15 \%$, escolaridade $2,26 \%$, paridade $1,80 \%$, gestação múltipla $0,00 \%$, cesárea eletiva $3,91 \%$, indução $21,71 \%$, cesárea prévia $5,47 \%$, pré-natal $2,18 \%$, tipo de parto $0,11 \%$. 
Do total de 23.507 partos ocorridos em maternidades com leitos $100 \%$ públicos ou $100 \%$ privados, dados referentes à classificação pelos critérios de Robson não estavam disponíveis para $7,8 \%$ dos partos públicos e $7,1 \%$ dos partos privados. A taxa global de cesariana foi de $66,5 \%$ e mostrou-se mais elevada entre as mulheres tendo parto no sistema privado, comparadas às do sistema público, $89,2 \%$ e $45,7 \%$ respectivamente $(p<0,001)$.

As mulheres que tiveram parto no setor privado apresentaram proporções significativamente mais elevadas $(p<0,001)$ nos grupos de maior idade, com maior nível de escolaridade, maior proporção de cor da pele branca, casadas ou em união estável e apresentaram uma maior frequência ao pré-natal. O setor público apresentou uma maior proporção de multíparas $(55,1 \%$ vs. 47,4\%; $p<0,001)$ e uma proporção menor de mulheres com cesariana prévia $(21,2 \%$ vs. $28,7 \%$; $p<0,001)$. O uso da indução foi três vezes mais comum nas mulheres do setor público (30,0\% vs $8,9 \% ; p<0,01)$, resultado em sintonia com cesarianas eletivas, sendo três vezes mais frequentes entre as mulheres do setor privado (22,5\% vs $65,4 \%$; $p<0,001)$. Por outro lado, não foram encontradas diferenças significativas na proporção de partos gemelares ao comparar os dois setores classificados pela forma de pagamento com proporções de 1,7\% no setor público e 1,9\% no privado $(p=0,41)$.

Do total dos 56.632 partos analisados no presente estudo, 11.294 (20\%) ocorreram em maternidades em que $100 \%$ dos leitos eram públicos; 10.434 (18,4\%) em maternidades com $100 \%$ dos leitos privados e 34.877 (61,6\%) em maternidades classificados como mistas, por apresentarem diferentes proporções de leitos públicos/privados.

A tabela 4 apresenta a distribuição dos partos e as taxas de cesariana de acordo com o SCR, estratificados por forma de pagamento pelo parto, incluídos apenas os hospitais/ maternidades com $100 \%$ dos leitos públicos ou $100 \%$ privados.

Tabela 4. Distribuição dos partos e das taxas de cesariana de acordo com Sistema de Classificação de Robson (SCR) estratificados por forma de pagamento pelo parto (sistemas público e privado). Santa Catarina, 2012.

\begin{tabular}{|c|c|c|c|c|c|c|c|c|c|}
\hline & & \multicolumn{4}{|c|}{ Público (SUS) } & \multicolumn{4}{|c|}{ Privado } \\
\hline \multicolumn{2}{|c|}{ GRUPOS DO SCR } & \multicolumn{2}{|c|}{ Cesariana } & \multicolumn{2}{|c|}{ Todos partos } & \multicolumn{2}{|c|}{ Cesariana } & \multicolumn{2}{|c|}{ Todos partos } \\
\hline & & $\mathbf{n}$ & (\%) & $\mathrm{n}$ & (\%) & $\mathbf{n}$ & (\%) & $\mathrm{n}$ & (\%) \\
\hline 1 & (Pri; Un; Cf; $\geq 37 ;$ Esp) & 587 & 32,2 & 1824 & 16,2 & 984 & 82,2 & 1197 & 11,5 \\
\hline 2 & (Pri; Un; Cf; $\geq 37 ;$ Ind/El) & 1442 & 56,6 & 2548 & 22,6 & 3481 & 93,4 & 3728 & 35,7 \\
\hline 3 & (Mt; Un; Cf; $\geq 37 ; \mathrm{Esp} ; \mathrm{Scp}$ ) & 152 & 8,4 & 1803 & 15,9 & 185 & 43,5 & 425 & 4,1 \\
\hline 4 & (Mt; Un; Cf; $\geq 37 ;$ Ind/El; Scp) & 332 & 28,5 & 1164 & 10,3 & 609 & 71,0 & 858 & 8,2 \\
\hline 5 & (Mt; Un; Cf; $\geq 37 ; \mathrm{Ccp})$ & 1543 & 77,7 & 1985 & 17,6 & 2513 & 98,0 & 2565 & 24,6 \\
\hline 6 & (Pri; Un; Pv) & 155 & 77,7 & 191 & 1,7 & 284 & 95,0 & 299 & 2,9 \\
\hline 7 & (Mt; Un; Pv;) & 186 & 75,0 & 248 & 2,2 & 166 & 90,2 & 184 & 1,8 \\
\hline 8 & (Todas; Mtp) & 167 & 79,1 & 211 & 1,9 & 206 & 97,6 & 211 & 2,0 \\
\hline 9 & (Todas; Un; Tv/ Ob) & 21 & 95,5 & 22 & 0,2 & 45 & 100,0 & 45 & 0,4 \\
\hline 10 & (Todas; Un; Cf; <37) & 580 & 44,7 & 1298 & 11,5 & 806 & 87,4 & 922 & 8,8 \\
\hline Total & & 5165 & 45,7 & 11294 & 100,0 & 9279 & 88,9 & 10434 & 100,0 \\
\hline
\end{tabular}

SUS - Sistema Único de Saúde; Pri (primípara); Mt (multípara); Un (gestação única); Mtp (gestação múltipla); Cf (cefálica); Pv (pélvica); Tv/ Ob (transversa ou obliqua); Ind/El (indução ou cesariana eletiva); Esp (espontâneo); <37(menos de 37 semanas de gestação); $\geq 37$ (37 ou mais semanas de gestação); Scp (sem cesariana prévia); Ccp (com cesariana prévia); todas (todas as mulheres). Dados não obtidos para forma de pagamento para $7,48 \%$ das cesarianas.

Ao observar os grupos 1 e 3, considerados de menor risco para cesariana, percebe-se que a proporção de parturientes nesses grupos combinados foi duas vezes mais comum no sistema público, comparado ao privado. Entretanto, as taxas de cesariana mostraram-se, significativamente, mais elevadas $(p<0,001)$ entre as mulheres do grupo 1 que tiveram parto no sistema privado $(82,2 \%)$, comparadas às usuárias do sistema público (32,2\%).

Observou-se ainda, entre as multíparas do grupo 3, que aparece um contraste importante ao comparar os dois sistemas de pagamento pelo tipo de parto, com taxas de cesariana, significativamente, mais baixas $(p<0,01)$ entre as mulheres do sistema público $(8,4 \%)$ comparadas às mulheres com o mesmo perfil no sistema privado $(43,5 \%)$.

Além disso, no sistema privado, 35,7\% dos partos foram representados pelo grupo 2 , enquanto a proporção das mulheres com as mesmas características, tendo parto no sistema público, foi significativamente menor $(22,6 \%)$. Diferenças no impacto 
nas taxas de cesarianas, ao comparar esses dois sistemas de pagamento, chamam à atenção, com $88,9 \%$ entre as usuárias do sistema privado contra $45,7 \%$ no sistema público $(p<0,001)$.

\section{DISCUSSÃO}

De acordo com dados do SINASC, foram registrados 88.926 nascidos vivos em 2012 para o estado de Santa Catarina. Um total de 56.632 nascimentos ocorreu nas 61 maternidades selecionadas para este estudo. A taxa geral de cesáreas nessa população foi de $60,7 \%$ e, no mesmo ano, a taxa de cesáreas para o estado como um todo foi de $60,74 \%{ }^{19}$. As taxas de cesariana foram duas vezes mais elevadas entre as mulheres cobertas pelo sistema privado $(88,9 \%)$, comparadas àquelas que tiveram parto no setor público $(45,7 \%)$. Os grupos $2,4,5$ e 10 foram os que apresentaram maior impacto nas taxas gerais de cesariana, responsáveis por $76,9 \%$ de todas as cesarianas. Todas essas diferenças apresentaram significância estatística.

Os dados do SINASC para a Região Sul do Brasil são reconhecidos como de boa confiabilidade, principalmente quando se compara a outras regiões do país ${ }^{20}$. A análise nos 61 estabelecimentos selecionados encontra-se em concordância com a taxa geral de cesarianas para Santa Catarina no ano do estudo, o que dá suporte à representatividade da amostra. Ainda que na classificação geral não se tenha separado indução e cesariana eletiva, mantendo o estudo fiel à classificação tradicional de Robson, examinou-se a cesariana eletiva como fator independentemente associado à forma de pagamento. Os dados no SINASC não permitem classificar as mulheres quanto ao risco obstétrico, e o SCR não inclui idade materna, dois fatores potencialmente associados à chance de cesariana. Entretanto, no mesmo tempo em que seria lúcido esperar uma maior proporção de risco obstétrico entre as mulheres com menor padrão socioeconômico, ou seja, aquelas que tiveram parto no sistema público, como já mostrado em outros estudos ${ }^{6,21}$, eventuais discrepâncias na distribuição dessas variáveis não seriam suficientes para explicar a magnitude das diferenças encontradas nas taxas de cesariana entre os dois sistemas de pagamento.

As taxas de cesariana em Santa Catarina encontradas neste estudo são superiores aos 51,9\% reportados por um recente estudo de base populacional ${ }^{21}$, assim como aquelas referidas internacionalmente como as mais elevadas do mundo ${ }^{22}$.

Nesse estudo, resultados baseados na classificação de Robson mostraram que as gestações únicas, cefálicas e a termo, tanto entre primíparas, quanto multíparas (grupos 1 a 5), contribuíram com mais de $80 \%$ da proporção total de cesarianas, e resultados semelhantes são apontados por outros artigos ${ }^{23-26}$; enquanto na ausência de indução, cesariana prévia ou eletiva (grupos 1 e 3) perfizeram apenas $25,5 \%$ de todos os partos e $14 \%$ de todas as cesarianas. Por outro lado, o risco de cesariana aumentou de maneira importante entre as mulheres, tendo parto com feto único, cefálico e a termo, ao associar o uso de indução ou presença de cesariana prévia (grupos 2, 4 e 5), passando a contribuir com $67,4 \%$ das cesarianas. Os resultados apresentados estão em concordância com diversos estudos em nível global ${ }^{26,27}$. Ademais, os resultados mostram que a indução e a cesariana prévia foram responsáveis por mais de $95 \%$ do excesso relativo no número de cesarianas e por um aumento de até quatro vezes nas proporções de cesariana quando comparados aos grupos com ausência desses fatores.

Apesar de as multíparas com gestação gemelar e multíparas com feto em apresentação não cefálica terem mostrado um excesso nas taxas de cesariana, quatro a cinco vezes mais elevadas, quando comparadas àquelas que tiveram fetos únicos e cefálicos, esses grupos foram responsáveis por um excesso relativo de apenas $5 \%$ no número de cesárias atribuíveis a estes fatores, gestação múltipla e apresentação.

Ao considerar apenas as primíparas com feto cefálico, parto a termo e espontâneo (grupos 1 e 2), observa-se que elas foram responsáveis por $37,5 \%$ de todas as cesarianas, resultados muito próximos dos $39 \%$ encontrados em estudo conduzido em nível nacional ${ }^{21}$ e dos $36,4 \%$ na América Latina ${ }^{27}$. Da mesma forma, multíparas sem cesariana prévia com feto cefálico e gravidez a termo (grupo 3 e 4), quando combinadas, contribuíram com $14,2 \%$ das cesarianas. Dessa forma, a proporção quase triplicou ao migrar, em função da utilização de indução ou cesariana eletiva, do grupo 3 para o 4 . Esses resultados se mostram bem mais elevados do que os reportados por estudos em outros países ${ }^{23,24,26}$.

Os achados sugerem uma crescente utilização da cesariana sem indicação médica estrita nos grupos considerados de baixo risco potencial para cesárea, como em gestações com feto único, a termo, cefálico e com trabalho de parto espontâneo, quando comparados a resultados de outros países ${ }^{23-26}$. Resultados da análise a partir do inquérito da OMS em 21 países $^{16}$ levaram os autores a concluir que a evolução temporal das taxas de cesariana sugere uma contribuição crescente das cesarianas sem indicação médica e daquelas decididas, eletivamente, antes do início do trabalho de parto. No atual estudo, $43 \%$ das cesarianas ocorreram de forma eletiva, proporção maior do que a encontrada para o Brasil no mesmo ano ${ }^{21}$.

Os resultados mostram que o grupo 5, composto pelas multíparas com feto único, cefálico, a termo e que tiveram cesárea prévia, foi responsável pela maior contribuição nas taxas de cesariana em Santa Catarina. Isso chama a atenção para denominado "efeito dominó" levantado pela $\mathrm{OMS}^{16,17}$, que corresponde a taxas crescentes de cesárea entre as primíparas e consequente acúmulo de mulheres com cesariana prévia, potencialmente sujeitas a um maior risco de uma nova cesariana $^{28}$. No contexto da prática obstétrica no Brasil, em que uma cesariana prévia decreta, de maneira importante, a probabilidade de uma nova cesariana ${ }^{11}$, é especialmente preocupante notar que as multíparas de baixo risco e cesárea prévia, as quais apresentaram taxas de cesariana altamente elevadas, aproximando-se dos $90 \%$, constituem mais de $20 \%$ da população de parturientes, tornando esse grupo responsável 
por quase $30 \%$ de todas cesarianas. Esses resultados estão em consonância com os 30,8\% reportados para o Brasil como um todo ${ }^{21}$ assim como pelo inquérito da OMS para América Latina no qual o grupo 5 contribuiu com $26,7 \%$ de todas as cesarianas ${ }^{27}$.

Por último, os grupos com apresentação não cefálica ou com partos múltiplos (grupos 6, 7, 8 e 9) perfizeram $9,1 \%$ de todas as cesarianas, número em estreita relação com os $8,9 \%$ encontrados no estudo "Nascer no Brasil"22 e inferior aos resultados encontrados para a América Latina, Europa, Canadá e Estados Unidos ${ }^{23-27}$.

Neste estudo, as mulheres que tiveram parto no setor privado eram, em maior proporção, brancas, pertenciam às faixas etárias mais elevadas, com maior nível de escolaridade, características frequentemente associadas com maior utilização da cesariana ${ }^{6,10}$.

As taxas de cesariana foram, praticamente, o dobro no setor privado (88,9\%), quando comparadas aos $45,7 \%$ encontrados para o setor público, tendo sido mais elevadas no setor privado para todos os grupos do SCR. Ainda que a participação dos dois grupos considerados como de menor potencial para cesariana (grupos 1 e 3) tenha sido mais elevada no setor público, ou seja, aquelas sem os fatores potencialmente associados a uma maior chance de cesariana (indução, cesariana eletiva ou cesariana prévia), as taxas de cesariana foram até 5 vezes mais elevadas entre essas mulheres quando o parto ocorreu no setor privado. No grupo 3, o qual apresentou a menor proporção de cesariana da amostra geral, a taxa de cesariana foi de $8,4 \%$ no setor público, comparada a $43,5 \%$ no setor privado.

As taxas de cesariana entre as multíparas com cesariana prévia (grupo 5), ainda que muito elevadas em ambos os setores, alcançaram quase $100 \%$ das mulheres no setor privado. Esses resultados estão em sintonia com os resultados para o Brasil como um todo, de acordo com forma de pagamento pelo parto $^{22}$.

Resultados da Pesquisa Nacional de Demografia em Saúde (PNDS) $^{29}$ mostraram que $46 \%$ das mulheres entrevistadas referiram ter sua cesariana decidida eletivamente. No presente estudo, $43 \%$ das cesarianas ocorreram de forma eletiva, embora essa proporção seja três vezes mais elevada entre as mulheres que tiveram parto privado $(65,4 \%)$, comparadas àquelas que tiveram parto no setor público $(22,5 \%)$.

A baixa utilização da indução no sistema privado pode ser explicada em grande parte pela alta concentração de multíparas com cesariana prévia no grupo privado e pelos mais de $64 \%$ dos partos decididos eletivamente no mesmo sistema. As taxas de indução encontradas no estudo foram mais elevadas, tanto no sistema público, quanto no privado, do que aquelas descritas pelo inquérito conduzido no Brasil ${ }^{22}$ e também quando comparadas aos resultados de outros países na Europa ${ }^{23,24}$ e América Latina ${ }^{27}$.

\section{CONCLUSÃO}

As altas taxas de cesariana encontradas são preocupantes, principalmente pelos potenciais efeitos adversos para as mães e seus recém-nascidos. Além disso, chama à atenção o aparente paradoxo de taxas bem mais elevadas entre as mulheres do sistema privado, exatamente aquelas com um perfil socioeconômico privilegiado, no qual poderiam ser esperadas menores taxas. Taxas elevadas entre as primíparas antecipam o efeito cumulativo da cesariana prévia entre as multíparas. A grande proporção de cesarianas eletivas somada à opção pela indução em detrimento do manejo ativo do trabalho de parto, principalmente no setor privado, aponta para um grande número de cesarianas realizadas sem indicações estritamente médicas para o procedimento.

Resultados corroboram aqueles de outros estudos, apontando para a influência da forma de pagamento, sugerindo diferenças na flexibilização da conduta médica e uma maior permeabilidade do obstetra ao decidir-se pela cesariana em face das indicações relativas para o procedimento.

Os resultados buscam contribuir para o melhor entendimento da questão das altas taxas de cesariana, permitindo priorizar áreas de intervenção e impacto. O objeto central do presente estudo, referente à aplicação do SCR no entendimento dos determinantes das taxas de cesariana no estado de Santa Catarina não foi até aqui explorado por nenhum outro estudo.

\section{REFERÊNCIAS}

1. Betrán A, Ye J, Moller A, Zhang J, Gülmezoglu A, Torloni M. The Increasing Trend in Caesarean Section Rates: Global, Regional and National Estimates: 1990-2014. PloS One. 2016 Feb;11(2):e0148343. doi: 10.1371/journal. pone. 0148343 .

2. Brasil. Ministério da Saúde. Sistema de Informação de Nascidos Vivos (SINASC). Cadernos de Informação de Saúde [Internet]. [acesso em 10 Abril 2018]. Disponível em: http://tabnet.datasus.gov.br/cgi/tabcgi.exe?\%20sinasc/ cnv/nvsc.def

3. World Health Organization. WHO statement on caesarean section rates. Genebra: WHO; 2015. [acesso em 2018 Jun 14] Disponível em: http://apps. who.int/iris/bitstream/10665/161442/1/WHO_RHR_15.02_eng.pdf?ua=1.
4. Sobhy S, Arroyo-Manzano D, Murugesu N, Karthikeyan G, Kumar V, Kaur I et al. Maternal and perinatal mortality and complications associated with caesarean section in low-income and middle-income countries: A systematic review and meta-analysis. Lancet. 2019 May; 393(10184):1973-1982. doi: 10.1016/S0140$6736(18) 32386-9$

5. Mascarello K, Matijasevich A, Santos I, Silveira M. Complicações puerperais precoces e tardias associadas à via de parto em uma coorte no Brasil. Rev Bras Epidemiol. 2018 Ago; 21: e180010. doi: http://dx.doi.org/10.1590/1980549720180010

6. Freitas PF, Savi E. Desigualdades sociais nas complicações da cesariana: uma análise hierarquizada. Cad Saúde Pública. 2011 Out; 27(10):2009-20. doi: 


\section{Sistema de Classificação de Robson na avaliação das taxas de cesariana}

http://dx.doi.org/10.1590/S0102-311X2011001000014.

7.Hopkins K, Amaral EFL, Mourão ANM. The impact of payment source and hospital type on rising cesarean section rates in Brazil, 1998 to 2008. Birth. 2014 Jun; 41(2): 169-77. doi: 10.1111/birt.12106.

8. Ramires de Jesus G, Ramires de Jesus N, Peixoto FM Filho, Lobato G. Caesarean rates in Brazil: What is involved? BJOG. 2015 Apr; 122(5): 606-9. doi: 10.1111/1471-0528.13119.

9. Leal MC, Pereira APE, Domingues RMSM, Theme MM Filha, Dias MAB, Nakamura-Pereira $\mathrm{M}$, et al. Obstetric interventions during labor and childbirth in Brazilian low-risk women. Cad Saúde Pública. 2014; 30(supl 1): S17-32. doi: http://dx.doi.org/10.1590/0102-311X00151513.

10. Rattner D, Moura EC. Nascimentos no Brasil: associação do tipo de parto com variáveis temporais e sociodemográficas. Rev Bras Saúde Mater Infant. 2016;1 6(1):39-47. doi: http://dx.doi.org/10.1590/1806-93042016000100005.

11. Freitas PF, Fernandes TMB. Associação entre fatores institucionais, perfil da assistência ao parto e as taxas de cesariana em Santa Catarina. Rev Bras Epidemiol 2016; 19(3): 525-38. doi: http://dx.doi.org/10.1590/19805497201600030005.

12. Robson MS. Classification of caesarean sections. Fetal Matern Med Rev. 2001;12(1):23-39

13. Torloni M, Betran A, Souza J, Widmer M, Allen T, Gulmezoglu M et al. Classifications for cesarean section: A Systematic Review. PloS ONE. 2011;6(1):e14566

14. Boatin AA, Cullinane F, Torloni MR, Betrán AP. Audit and feedback using the Robson classification to reduce caesarean section rates: a systematic review. BJOG. 2018; 125(1): 36-42. doi: 10.1111/1471-0528.14774.

15. Betrán AP, Vindevoghel N, Souza JP, Gülmezoglu AM, Torloni MR. A systematic review of the Robson classification for caesarean Section: What works, doesn't work and how to Improve It. PloS ONE. 2014 Jun; 9(6): e97769. doi: 10.1371/journal.pone.0097769.

16. Vogel JP, Betrán AP, Vindevoghel N, Souza JP, Torloni MR, Zhang J, et al. Use of the Robson classification to assess caesarean section trends in 21 countries: a secondary analysis of two WHO multicountry surveys. Lancet Glob Health. 2015 May; 3(5): e260-70. doi: 10.1016/S2214-109X(15)70094-X.

17. Ministério da Saúde. Portal da Saúde. 2017 [acesso em 6 Mar 2017] Disponível em: http://www2.datasus.gov.br/DATASUS/index. php?area $=0901 \&$ item $=1 \&$ acao $=28$.

18. Brasil. Ministério da Saúde. Cadastro Nacional de Estabelecimentos de Saúde (CNES). 2018 [ acesso em 22 Jan 2018] Disponível em: http://cnes. datasus.gov.br/pages/estabelecimentos/consulta.jsp.
19. Brasil. Ministério da Saúde. Sistema de Informação de Nascidos Vivos (SINASC). Cadernos de Informação de Saúde [Internet]. 2018 [acesso em 10 April 2018]. Disponível em: http://tabnet.datasus.gov.br/cgi/tabcgi.exe? sinasc/ cnv/nvsc.def

20. Ministério da Saúde (BR). Consolidação de Informações sobre os Nascidos Vivos - 2011. 2013. [Internet] Disponível em http://tabnet.datasus.gov.br/cgi/ sinasc/Consolida_Sinasc_2011.pdf

21. Nakamura-Pereira M, Carmo Leal M, Esteves-Pereira AP, Domingues RM, Torres JA, Dias MA, et al. Use of Robson classification to assess cesarean section rate in Brazil: The role of source of payment for childbirth. Reprod Health. 2016 Oct;13(S3):128.

22. World Health Organization. Global Health Observatory Data Repository. 2015 [acesso em 14 Jun 2018] http://apps.who.int/gho/data/view main.1630?lang=en.

23. Le Ray C, Blondel B, Prunet C, Khireddine I, Deneux-Tharaux C, Goffinet F. Stabilising the caesarean rate: Which target population? BJOG. 2015 Apr; 122(5):690-9. doi: 10.1111/1471-0528.13199.

24. Zhang J, Geerts C, Hukkelhoven C, Offerhaus P, Zwart J, de Jonge A. Caesarean section rates in subgroups of women and perinatal outcomes. BJOG. 2016 Apr;123(5):754-61. doi: 10.1111/1471-0528.13520.

25. Hehir M, Ananth C, Siddiq Z, Flood K, Friedman A, D'Alton M. Cesarean delivery in the United States 2005 through 2014: a population-based analysis using the Robson 10-Group Classification System. Am J Obstet Gynecol. 2018 219(1): 105.e1-105.e11. doi: 10.1016/j.ajog.2018.04.012.

26. Roberge S, Dubé E, Blouin S, Chaillet N. Reporting Caesarean Delivery in Quebec Using the Robson Classification System. J Obstet Gynaecol Can. 2017 Mar; 39(3):152-156. doi: 10.1016/j.jogc.2016.10.010.

27. Betrán AP, Gulmezoglu AM, Robson M, Merialdi M, Souza JP, Wojdyla $D$, et al. WHO global survey on maternal and perinatal health in Latin America: classifying caesarean sections. Reprod Health. 2009 Oct; 6:18. doi: 10.1186/1742-4755-6-18

28. Nakamura-Pereira M, Esteves-Pereira A, Gama S, Leal M. Elective repeat cesarean delivery in women eligible for trial of labor in Brazil. Int Journal of Gyn \& Obst. 2018;143(3):351-359.

29. Lago TDG, Lima LP. Assistência à gestação, ao parto e ao puerpério: diferenciais regionais e desigualdades socioeconômicas. In: Ministério da Saúde (BR), Centro Brasileiro de Análise e Planejamento. Pesquisa Nacional de Demografia e Saúde da Criança e da Mulher - PNDS 2006: dimensões do processo reprodutivo e da saúde da criança. Brasília (DF): MS/CEBRAP; 2009; 8:151-70

\section{Como citar este artigo/How to cite this article:}

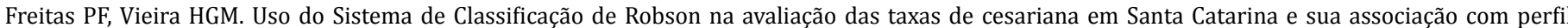
institucional. J Health Biol Sci. 2020; 8(1):1-9. 